\title{
COM \\ Embracing the future through science communication: the inaugural "Mr. Science" Science Communication Conference in China
}

\section{Reviewed \\ Conference}

Reviewed by

Abstract

Keywords

DOI
The inaugural "Mr. Science" Science Communication Conference, SUZHOU, CHINA, 9TH JULY 2021

\section{Guoyan Wang, Jingyi Han, Hepeng Jia and Yuqin Yang}

The inaugural "Mr. Science" Science Communication Conference was held in Suzhou, China on July 9, 2021. It was the largest Chinese conference on science communication study since the start of the 21st century. More than 260 scholars discussed the spirit and culture of science, science communication during the COVID-19 crisis, the public understanding of science, and the ethical aspects of science communication. The conference aimed to develop a system for researching science communication within China. This review outlines the content of the conference and summarizes the key trends in science communication research in China.

Public understanding of science and technology; Risk communication; Science communication: theory and models

https://doi.org/10.22323/2.20050605

Submitted: 20th August 2021

Accepted: 1st September 2021

Published: 22nd September 2021

"Mr. Science" is a concept derived from the New Culture Movement in 1915, when China introduced science from the west for the purposes of development [Schmalzer, 2021]. It connotes the culture and spirit of scientific inquiry.

The inaugural Suzhou "Mr. Science" Science Communication Conference was organized by the School of Communication at Soochow University. It was the largest Chinese conference on science communication study since the start of the 21 st century and it featured more than 260 science communication scholars and scientists. Totally 67 research items were presented during the plenary session (Figure 1) and the six sub-forums. Jenny Metcalfe, President of the network for the Public Communication of Science and Technology (PCST), delivered a speech at the opening ceremony in which she congratulated Soochow University on the establishment of the Science Communication Research Center (SCRC). Qide Han, a researcher and Honorary Chairman of the China Association for Science and 


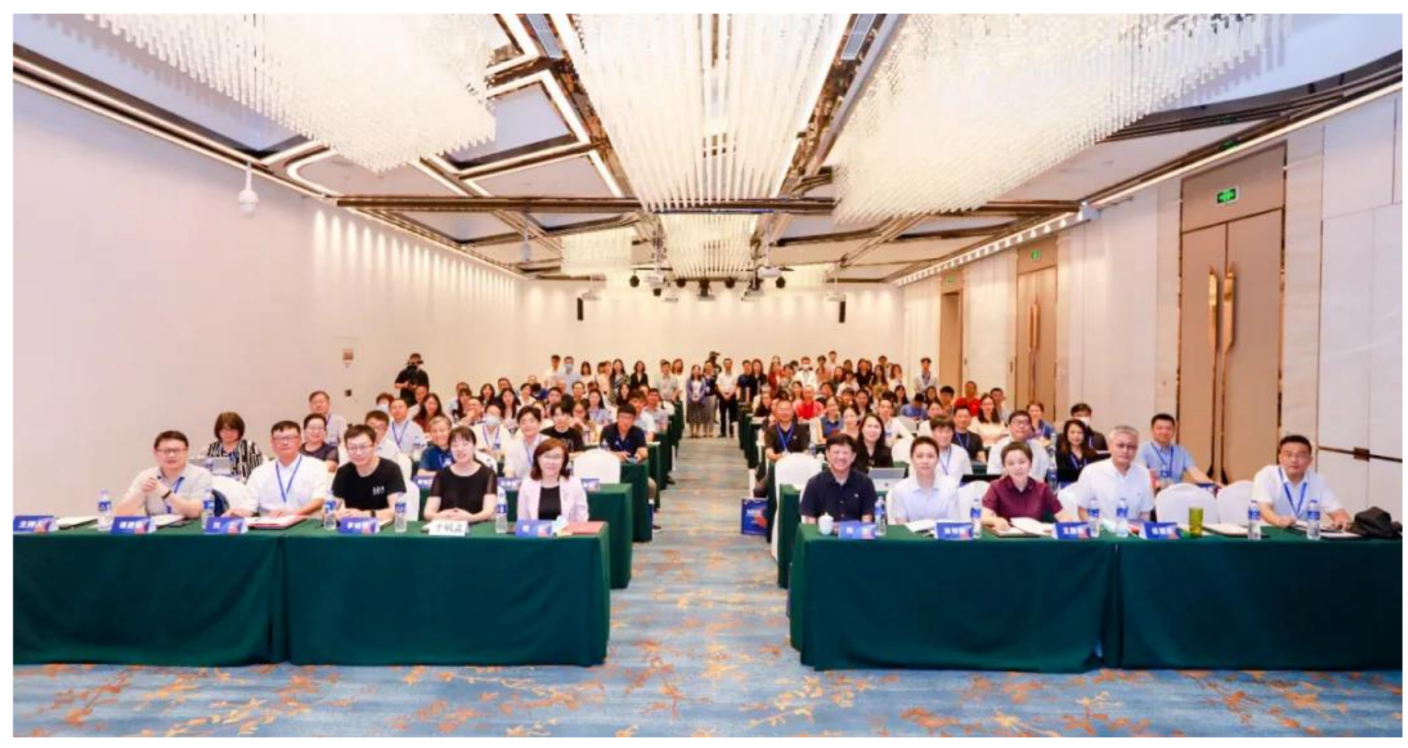

Figure 1. The main venue, Suzhou (c) the Science Communication Research Center at Soochow University.

Technology (CAST), proposed the following slogan for the conference: "Embracing the Future through Science Communication".

The conference reflected on how best to promote the spirit and culture of science. Fujun Ren, the President of the National Academy of Innovation Strategy for CAST, stated that the social image of Chinese scientists is closely linked to their social responsibilities. Therefore, they are constantly being shaped by changes in society. He also summarized the necessary elements required to communicate science through effective storytelling [Ren, Li and Liu, 2021]. Researcher Lianggang Ji of the Zhejiang Science Communication Center believes that the scientific spirit can reform society. He pointed out that there are currently several problems in China, such as a lack of infrastructure for promoting and disseminating science, as well as a lack of social atmosphere for promoting the culture of science. He argued that it was necessary to find practical ways of promoting and institutionalizing the spirit of science. Meanwhile, Professor Jianxun Chu from the University of Science and Technology of China (USTC) argued from a historical perspective that the true value of Mr. Science lies in its ability to communicate science. He also noted that the communication of science involves the distribution of scientific knowledge, subject to certain ethical considerations [Medvecky, 2018]. Professor Guoyu Wang from Fudan University also pointed to the ethical considerations. Starting from the concept of dual-use science (which refers to the combination of risk and freedom), she noted the importance of freedom in scientific publishing, while also recommending mechanisms for supervising how science is communicated to the public. Professor Jie Liu from Huazhong University of Science and Technology argued that the spirit of science is both creative and destructive. The processes of questioning and criticizing inherent in scientific inquiry may have an impact on the established social order. As such, it is important to think carefully before communicating scientific findings to the wider public. Researcher Chunliang Fan from the Institute of Science and Development concluded by arguing that promoting the spirit of science requires patriotism, solidarity, collaboration, and a desire for truth. These values must be cultivated in the next generation. 
Two leaders of Soochow University's Science Communication Research Center, Professor Guoyan Wang and Professor Hepeng Jia, shared recent research into the communication of science during public crises. Professor Wang's group shared a series of studies on the relationship between the public, policymaking and scientific research during the pandemic. They found that strict mobility restrictions were more likely to cause a surge in population outflow from the epidemic area in the short term. By contrast, they found that mobility restrictions were positively related to the stringency of policies in the long term. Meanwhile, they found that public behaviour proceeded in three phases, which is typical of an epidemic. They focused on the co-evolution between research into COVID-19 and China's policies, argued that this should have consequences for evidence-based decision-making in the future [Cheng et al., 2021]. Wang's group also tackled the controversial subject of genetic modification (GM). Their research reported that online cartoons about GM had contributed to the intensification of the debate around GM in China [Wang, Wang and Shen, 2021]. Professor Jia's group looked at the correlation between patriotism and the willingness of Chinese citizens to be vaccinated or work to combat COVID-19. The group found that these two variables (patriotism and anti-epidemic behavior) were significantly correlated [Jia and Luo, 2021]. A study of scientists' intentions when it came to communicating about science during the pandemic revealed that those intentions were closely related to their understanding of the moral value of science and their feelings towards their homeland. Professor Jia's group also studied the influence of conspiracy theories on people's behaviors regarding their health. They proposed that effective science communication would help to reduce the harmful impact of conspiracy theories.

When it comes to the public understanding of science, Professor Mingxin Zhang from Huazhong University of Science and Technology shared his study on the attitudes of the public regarding artificial intelligence (AI). He stated that the public's understanding and attitudes can change the nature of scientific and technological research, as well as influencing policymaking and industry applications. Professor Jianping He from Southwest University of Political Science and Law argued that the spread of misinformation damages any attempt to ensure successful science communication. In a study of the effects of misinformation on communicating information about healthcare to inner-city elderly people, she found that even those who were better educated tended to believe misinformation when they were unsure.

The conference coincided with the release of the National Science Literacy Action Plan (2021-2035), hereafter the 2021 Plan, a policy that will guide the development of national science communication over the next 15 years. Professor Li Liu from Tsinghua University pointed out that the 2021 Plan will help to ensure scientific literacy for all. Prof. Liu also states that a new measurement system adapted to the Chinese context should be established as soon as possible to remain comparable the international standards for evaluating scientific literacy in China. Professor Jianxun Chu also stated that it will be important to pay attention to issues like how the effects of science communication are evaluated and what new disciplines should be created in the field of science communication. Professor Jie Liu pointed out that the design of disciplines should be based on practice, and Professor Xiaojin $\mathrm{Gu}$ from Shenzhen University hoped that relevant textbooks will be published. Professor Rongting Zhou from USTC reminded the audience that science communication can also be studied from the perspective of science education. 


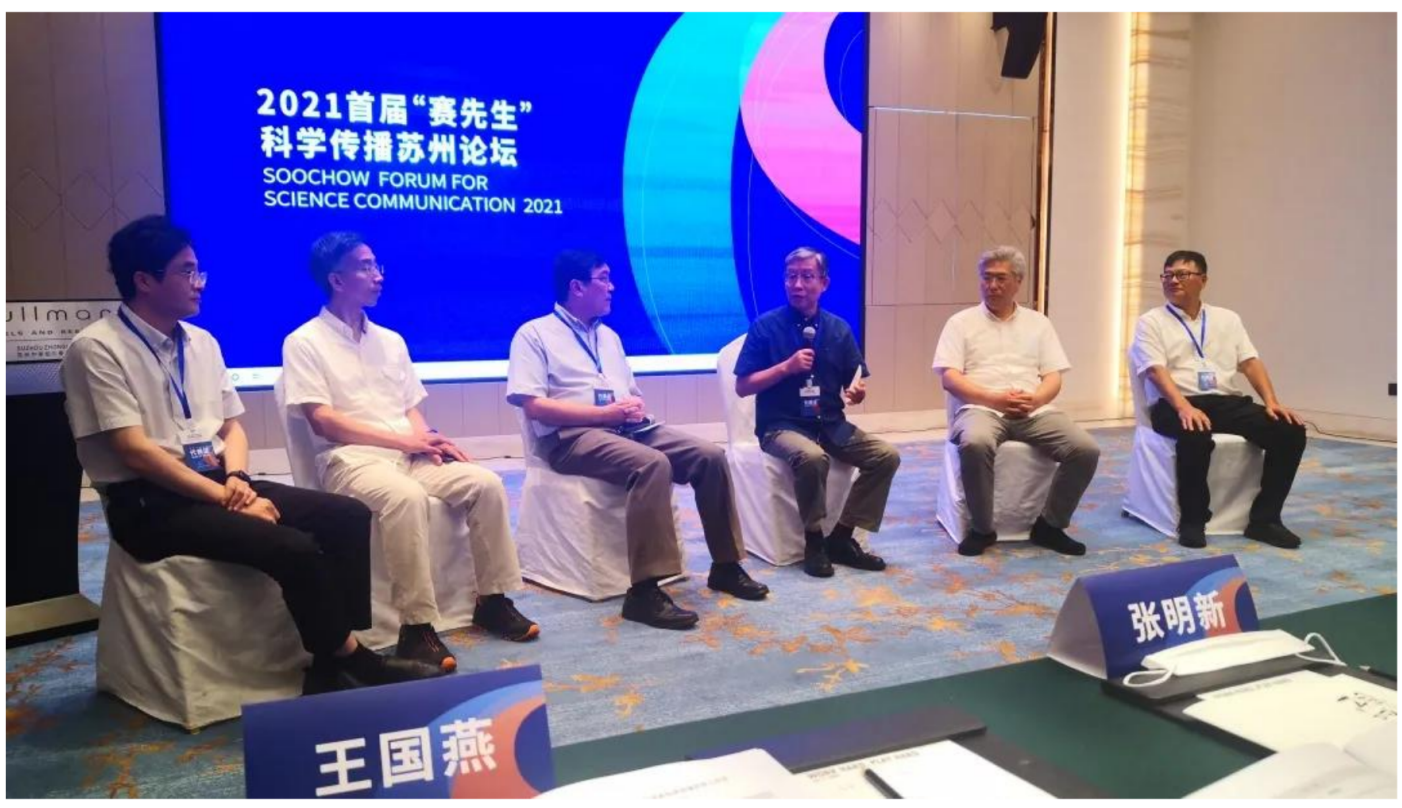

Figure 2. The round table discussion at the main conference (C) SCRC.

To that end, he also introduced the newly established Chinese journal: Science Communication and Science Education.

In the roundtable discussion session (Figure 2), Professor Xiaojin Gu, the host of the event, detailed how an important wave of research into science communication had occurred during the early 1980s and the mid-1990s. He stated that a new climax was currently in development, under the guidance of Soochow University, Tsinghua University, the University of Science and Technology of China, and Huazhong University of Science and Technology. Professors Li Liu, Cong Cao, Rongting Zhou, Jianbin Jin, and Baoguo Cui engaged in a heated debate on "the science communication research system in China". The panelists pointed out several directions for the future development of Chinese science communication research.

Alongside the main conference, there were two parallel workshops dedicated to discussing science communication research methods and practical skills. There were also six sub-forums, each of which looked at a specific topic within science communication: public crises; scientific innovation in the age of integrated media; interdisciplinary research in science communication; new trends in health communication; science fiction and science writing; and the communication of scientific achievements. In summary, the inaugural "Mr. Science" science communication conference in Suzhou was not only a milestone in Chinese research into science communication but also an important catalyst for future research in the field. 
Cheng, X., Tang, L., Zhou, M. and Wang, G. (2021). 'Coevolution of COVID-19 research and China's policies'. Health Research Policy and Systems 19, 121. https://doi.org/10.1186/s12961-021-00770-6.

Jia, H. and Luo, X. (2021). 'I wear a mask for my country: conspiracy theories, nationalism, and intention to adopt Covid-19 prevention behaviors at the later stage of pandemic control in China'. Health Communication. https://doi.org/10.1080/10410236.2021.1958982.

Medvecky, F. (2018). 'Fairness in knowing: science communication and epistemic justice'. Science and Engineering Ethics 24 (5), pp. 1393-1408. https://doi.org/10.1007/s11948-017-9977-0.

Ren, F.-J., Li, X. and Liu, X. (2021). 'Rethinking about telling the story of scientists and establishing the image of scientists in the context of anti-epidemic'. Studies in Dialectics of Nature 37 (06), pp. 65-72. https://doi.org/10.19484/j.cnki.1000-8934.2021.06.012.

Schmalzer, S. (2021). 'The global comrades of Mr. Democracy and Mr. Science: placing May Fourth in a transnational history of science activism'. East Asian Science, Technology and Society: an International Journal. https://doi.org/10.1080/18752160.2020.1868698.

Wang, G., Wang, L. and Shen, J. (2021). 'Food to politics: representations of genetically modified organisms in cartoons on the Internet in China'. Public Understanding of Science 30 (3), pp. 274-284.

https://doi.org/10.1177/0963662520983564.

\section{Authors}

Guoyan Wang is a professor in School of Communication, Soochow University, China. She is a scientific committee member of Public Communication of Science and Technology network (PCST). Her research has been published on Public Understanding of Science, Science Communication, Science as Culture, Journal of Informetrics, Leonardo and other leading journals. E-mail: gywang@suda.edu.cn.

Jingyi Han is a postgraduate student in School of Communication, Soochow University, China. E-mail: hanjing.yi711@163.com.

Hepeng Jia is a professor in School of Communication, Soochow University, China. E-mail: hpjia@suda.edu.cn.

Yuqin Yang is a postgraduate student in School of Communication, Soochow University, China. E-mail: 2278560545@qq.com.

\section{How to cite}

Wang, G., Han, J., Jia, H. and Yang, Y. (2021). 'Embracing the future through science communication: the inaugural "Mr. Science" Science Communication Conference in China'. JCOM 20 (05), R05. https:/ / doi.org/10.22323/2.20050605. 